\title{
AVO Analysis in Real Pre-Stack Seismic Data: A Powerful Tool for Fluid Identification During Petroleum Exploration
}

Mitchel Xavier (Halliburton) and Rodrigo S. Portugal (Halliburton)

Copyright 2019, SBGf - Sociedade Brasileira de Geofísica

This paper was prepared for presentation during the $16^{\text {th }}$ International Congress of the Brazilian Geophysical Society held in Rio de Janeiro, Brazil, 19-22 August 2019.

Contents of this paper were reviewed by the Technical Committee of the $16^{\text {th }}$ International Congress of the Brazilian Geophysical Society and do not necessarily represent any position of the SBGf, its officers or members. Electronic reproduction or storage of any part of this paper for commercial purposes without the written consent of the Brazilian Geophysical Society is prohibited.

\section{Abstract}

This work discusses the importance of analyzing the AVO behavior on pre-stack gathers to aid geoscientists with the identification of fluids and improve delimitation of the boundaries of hydrocarbon accumulation. To achieve this, a study was performed comprised of three AVO-based workflows applied to marine seismic data acquired from offshore Norway. The workflows are graphic visualization of amplitude versus offset curves, analysis of seismic volumes and cross-plots of intercept versus gradient, and interpretation of amplitude versus offset cube. In the case study, amplitude values increased significantly when the offset value increased, thus indicating the presence of gas. It is important to note that this conclusion can be made more accurately after completing the third workflow.

\section{Introduction}

It is well-known that the exploration process is an important and expensive stage of the petroleum industry. One of the most important investigations for the exploration process is so-called amplitude versus offset (AVO) analysis, which provides several applications for petroleum exploration including hydrocarbon detection, lithology identification, and fluid parameter analysis (CASTAGNA and BACKUS, 1993).

In summary, it is a seismic attribute extracted from prestack data in which the seismic amplitude variation is analyzed against the increasing of the offset, which is the distance between the source of the seismic wave and the receiver. Although the input of the original method is the pre-stack data, during the last two decades, operators and service companies have performed a simple version of the conventional AVO analysis, which uses only partial stacks (i.e., near, mid, far and ultra-far seismic volumes) to help the interpretation of the target area. When compared to the original AVO method, such simplification requires not only less computational resources and time but also less expertise from the interpreters.

This work proposes use of three AVO-based workflows applied to pre-stack marine seismic data. The study was performed using a dataset from offshore Norway. It relates to a hydrocarbon field located in the Norwegian Sea; water depth in the area is approximately 300 meters and the reservoir contains gas in Middle Jurassic sandstones of the Fangst Group. The reservoir depth is 2390 to 2490 meters (SHAHRI, 2013). Figure 1 shows a seismic section from a Kirchhoff time migrated seismic volume illustrating the top of Fangst Group reservoir and well trajectories.

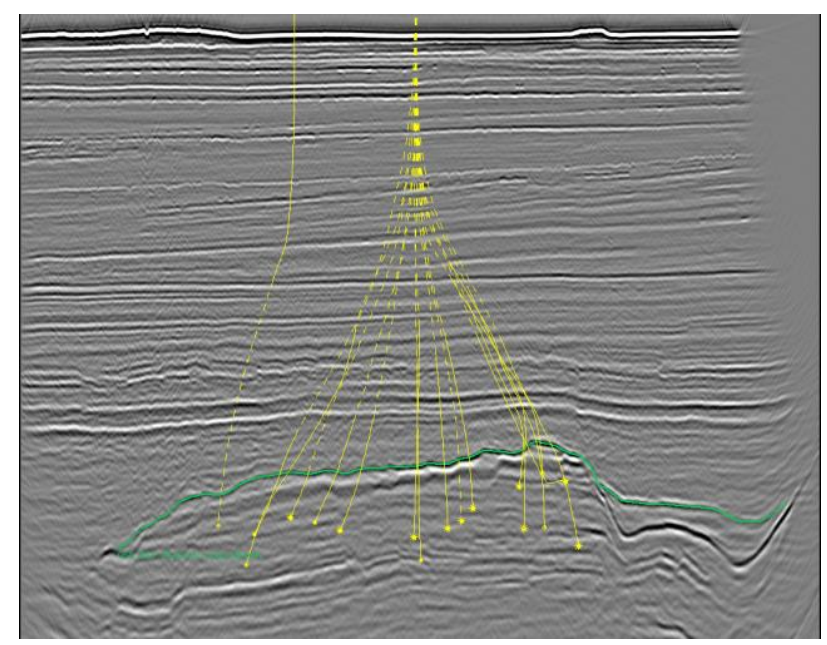

Figure 1: Seismic section from offshore Norway. The top of reservoir formation is indicated by the green horizon and, in yellow, are some trajectories of production and injection wells.

\section{Method}

The first workflow begins with interpreting a horizon on the pre-stack gathers. The corresponding post-stack horizon is used as a guide for an auto-tracking workflow to pick the horizon on the pre-stack gathers.

Then, for each gather, a curve is generated, which is the amplitudes for each offset value and all the gathers along the pre-stack horizon (Figure 2). Finally, after a quick screening through the gathers by analyzing the plots, it is relatively easy to identify the region that presents a particular amplitude variation with offset that indicates possible anomalies related to the presence of hydrocarbons (CASTAGNA and SWAN, 1997).

The second workflow involves two AVO attributes called intercept and gradient, which are respectively the estimated amplitude for the zero-offset cube and the rate of change of the amplitude with respect to offset. Basically, volumes of intercept and gradient are built from pre-stack seismic data. With such volumes (Figure 3 ), it is possible to generate a two-dimensional visualization of the anomalies (i.e., the anomalies can be identified at the 
level of the interpreted pre-stack target horizon), in such a way that their position spotted at the intercept and gradient maps matches the identified regions of the anomalies when the graphical plots were analyzed. After that, a crossplot between the intercept and gradient volumes is generated (Figure 4).

The last workflow is the generation of an offset amplitude volume, which is the result of the gathering of all graphical plots created in the first workflow, but rotated $90^{\circ}$ clockwise. If the plots are maintained as they were originally created, it is impossible to generate overlay graphics for comparison of the pre-stack amplitude cube and the post-stack seismic volume. This is because, for one common depth point (CDP), there are several values of amplitude, one value for each offset. However, when a $90^{\circ}$ rotation is applied, the offset axes move from the horizontal to the vertical direction, allowing the several values of amplitude, one value per offset per CDP, to be displayed along the proper CDP trace. After that, it is possible to overlay the volumes with additional control of opacity, which can highlight the anomalies of amplitude of the pre-stack cube. This technique allows the geoscientist to perform a global analysis by examining the post-stack seismic volume and the AVO anomalies extracted from the pre-stack seismic data simultaneously.

\section{Results}

Figure 2 shows the results after application of the first workflow. It is easy to see, qualitatively, that the amplitude absolute value increases with the offset along the horizon in red color, which represents the top of reservoir.

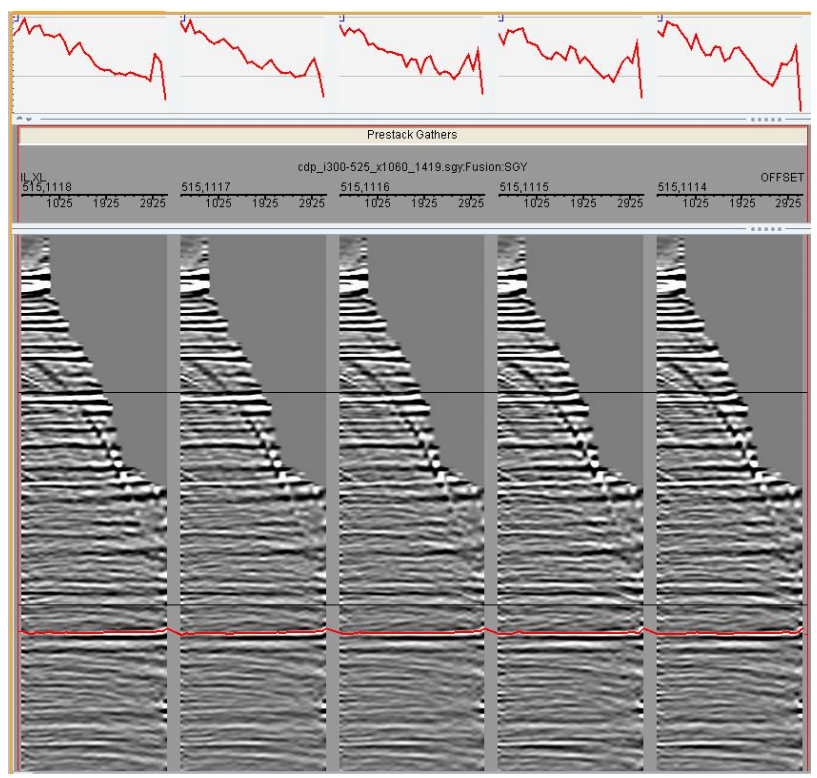

Figure 2: Pre-stack seismic data from offshore Norway. At the top of the figure amplitude vs. offset is graphically illustrated related to the values measured along the top of the reservoir horizon, in red in the pre-stack seismic shown in the lower part of the figure.
It worth mentioning that, depending on the goal of the study, such analysis of graphical plots can be enough to help mitigate some remaining doubt related to the exploration play, particularly when conventional AVO analysis does not provide the required answers. In Figure 3 can be found the results of the generated intercept and gradient volumes and Figure 4 shows the crossplot between them.

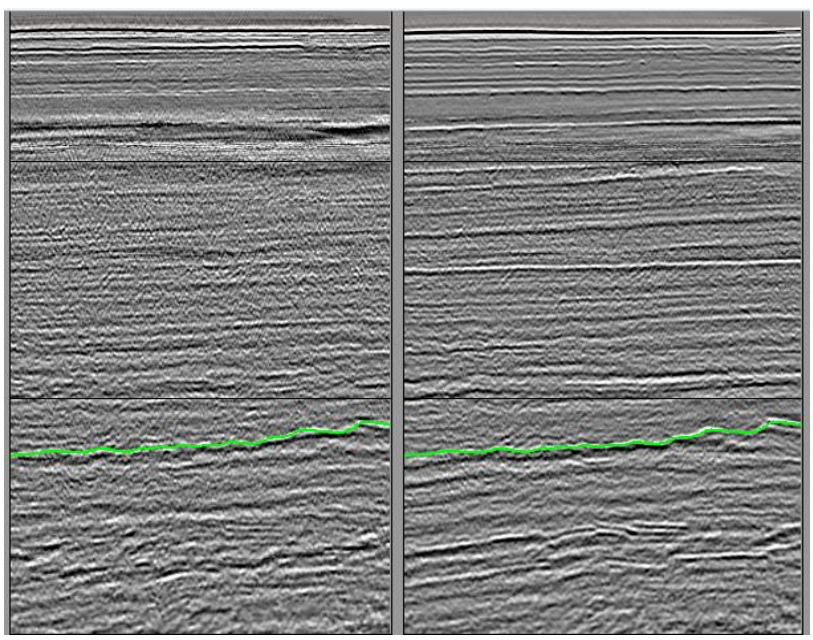

Figure 3: Intercept (right) and gradient (left) volumes calculated from pre-stack seismic data. The green horizon indicates the top of reservoir.

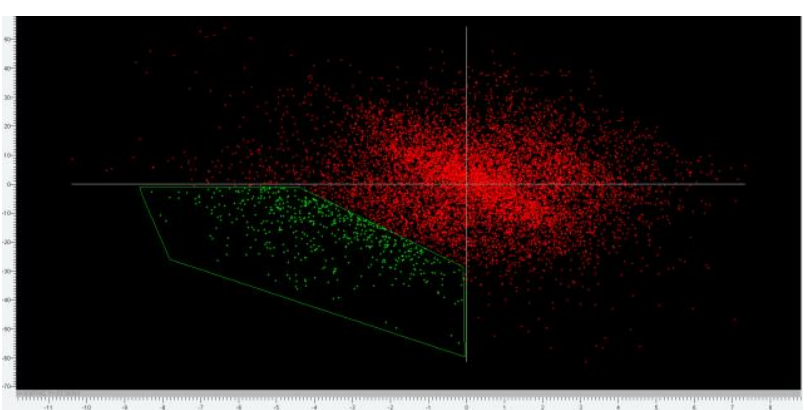

Figure 4: Crossplot between intercept and gradient volumes. The green polygon represents the selection of the AVO anomaly region.

For the case study, through the analysis of the crossplot, it was clear there were several points located in the region representing a Class III AVO anomaly (Zhang and Brown, 2001), which typically indicates the presence of gas. It is possible to see in Figure 5 the region with red dots in the seismic section, which corresponds to the selected points inside the green polygon in Figure 4. The majority of the red dots are at the top of reservoir. As previously discussed, the Fangst Group reservoir contains gas, confirming the validity of the results. 


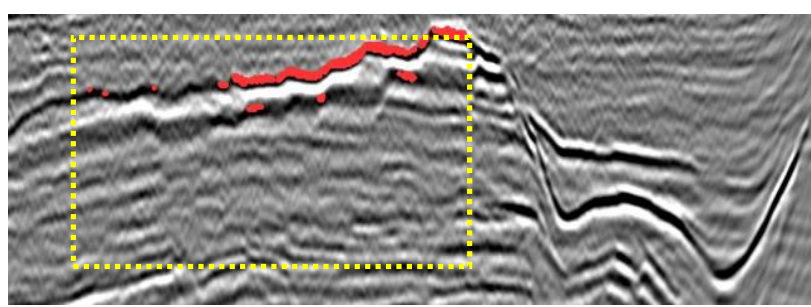

Figure 5: Reservoir region. The red dots represent the anomaly region related to the green polygon in the figure 4. The yellow dashed square corresponds to the selected area for the next step of the study.

The last workflow was only applied to the region inside the yellow dashed square (Figure 5). A zoom of such region is displayed in Figure 6.

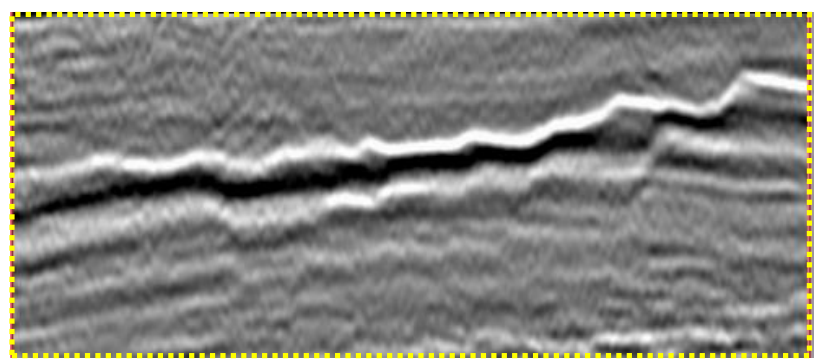

Figure 6: Zoom-in of the selected area where the amplitude cube will be calculated from pre-stack seismic.

A two-dimensional view of the amplitude cube is shown in Figure 7. The amplitude anomalies are illustrated on the left size of the Figure using arrows. The green color represents the amplitude with small absolute values. In some areas of the amplitude cube, it is easy to realize the strong change in amplitude absolute value. Thus, the AVO anomaly can also be identified in the amplitude cube.

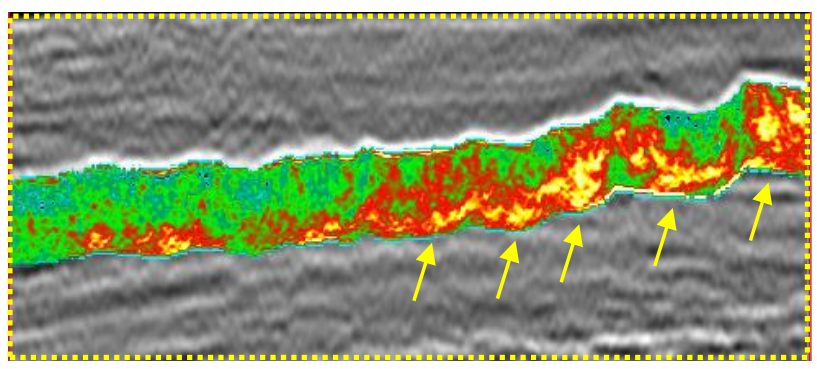

Figure 7: $A 2 D$ view of the amplitude cube hung on the top of the reservoir horizon.

\section{Conclusions}

After performing the proposed workflows in the case study, the visual analysis of graphical plots, intercept, and gradient map analyses, including crossplot generation and the volumetric comparison, it is safe to conclude a strong presence of gas in the area of interest. Additionally, it was easy to identify the boundaries of the potential reservoir. AVO analysis has contributed to the exploration process for the last two decades; but, the conventional and simple way of using partial stacks does not always provide reliable information to the geoscientist. With technology advances and new technical challenges, new methods to perform analysis of the pre-stack data are becoming increasingly more necessary and, sometimes, indispensable for AVO analysis as well as other types of studies, such as seismic inversion.

\section{Acknowledgments}

The authors thank Halliburton for providing the software, dataset, and support.

\section{References}

CASTAGNA, J.P. AND BACKUS, M.M. 1993. AVO analysis-tutorial and review in Castagna, J. and Backus, M.M., eds, Offset-dependent reflectivity. Theory and practice of AVO analysis: Soc. Expl. Geophys., 3-37.

CASTAGNA, J.P. AND SWAN, H.W. 1997. Principles of AVO crossplotting: AAPG Mid-Continent Section Meeting; Abstracts; AAPG Bulletin, 81, no. 8, 1348.

SHAHRI, S. A. G. 2013. Application of extended elastic impedance (EEI) to improve reservoir characterization. Master Thesis. Norwegian University of Science and Technology.

ZHANG, H., AND BROWN, R. J., 2001, A review of AVO analysis: CREWES Research Report, Vol. 13. 